\title{
Empirical analysis of RNA robustness and evolution using high-throughput sequencing of ribozyme reactions
}

\author{
Eric J. Hayden \\ Department of Biological Sciences \\ Biomolecular Sciences PhD Program \\ Boise State University, Boise, Idaho 83725 \\ phone: (208) 426-4625 \\ email:erichayden@boisestate.edu
}

\begin{abstract}
RNA molecules provide a realistic but tractable model of a genotype to phenotype relationship. This relationship has been extensively investigated computationally using secondary structure prediction algorithms. Enzymatic RNA molecules, or ribozymes, offer access to genotypic and phenotypic information in the laboratory. Advancements in high-throughput sequencing technologies have enabled the analysis of sequences in the lab that now rivals what can be accomplished computationally. This has motivated a resurgence of in vitro selection experiments and opened new doors for the analysis of the distribution of RNA functions in genotype space. A body of computational experiments has investigated the persistence of specific RNA structures despite changes in the primary sequence, and how this mutational robustness can promote adaptations. This article summarizes recent approaches that were designed to investigate the role of mutational robustness during the evolution of RNA molecules in the laboratory, and presents theoretical motivations, experimental methods and approaches to data analysis.
\end{abstract}




\section{Introduction}

As a model system to study evolution in the laboratory, ribozymes (enzymatic RNA molecules) have several desirable features that facilitate experimental control and analysis. Every individual RNA molecule contains both a genotype in its sequence of nucleotides, and a phenotype, defined as its structure or function. The standard tools of molecular biology (i.e. PCR, in vitro transcription, reverse transcription) can be used to replicate ribozymes. Sequence diversity can be introduced either during chemical synthesis or enzymatic replication to create vast populations of molecules, with population sizes typically ranging from several thousands up to $\sim 10^{15}$ molecules. Error-prone PCR conditions can be used during replication to alter the mutation supply rate. With control over population size and mutation rates, experiments can be designed to achieve rapid rates of evolution, or to match specific conditions of natural populations. The function of the ribozymes can be used to impose preferential replication of the most functional molecules, resulting in "the survival of the fittest" at the molecular level. Finally, changing variables such as temperature, $\mathrm{pH}$, salt concentrations, and substrate availability can control the physical and chemical environment. Through these manipulations, the selection pressure can be controlled, and the number of molecules that are allowed to replicate can be indirectly manipulated. Thus, the in vitro selection of RNA molecules maintains many of the forces of evolution found in natural systems, yet also allows experimental manipulation of these forces.

From a population genetics perspective, evolution is detected as the change in the genetic composition of a population over time. Changes in the composition 
are caused by the combined forces of evolution, including mutation, selection, and drift. Selection can be detected by sequencing genotypes over time, as high-fitness genotypes will increase in population frequency at the expense of lower fitness genotypes. Therefore, another major advantage of RNA molecules is that they can be converted to DNA and analyzed by sequencing. Changes in the composition of the RNA population over time can be observed. From these frequency changes, "fitness" values can be assigned. Note that researches that evolve protein enzymes, RNA enzymes (ribozymes) and aptamers often refer to relative activity as "protein fitness" or "RNA fitness" because this relative activity determines the probability of a molecule being present in subsequent populations. Classically, frequency measurements involved cloning populations of molecules followed by Sanger sequencing of individual molecules[1]. Now, very large numbers of individual molecules can be sequenced simultaneously using high-throughput sequencing platforms. This large number of sequences can be spread over several time points during the course of an evolution experiment, in order to observe the rise and fall of specific combinations of mutations. Alternatively, this large number of sequences can be used to achieve very deep sequencing of a single population before and after one round of selection[2,3]. This latter approach maximizes the diversity of populations that can be analyzed, as well as the range of fitness measurements, down to very low fitness individuals, but may not allow observation of interesting longer term dynamics[4].

In addition, RNA secondary structures have served as a realistic model phenotype that can be predicted and evolved computationally[5]. From such work 
has emerged the concept of genotype networks, defined as the genotypes that are accessible by small mutational steps and that maintain a given phenotype, such as a particular secondary structure in the case of RNA[6,7]. Many computationally explored RNA genotype networks span vast regions of sequence space[8]. RNA structures possess varying degrees of mutational robustness, defined as the ability to maintain a phenotype despite random mutations. For mutationally robust RNA structures, it is possible to make a very large number of consecutive nucleotide substitutions and maintain the same predicted secondary structure. Populations of RNA molecules can therefore harbor standing genetic variation while presenting unchanged phenotypes. However, while the sequences of a given genotype network all fold into the same minimum free energy structure, the new phenotypes that become accessible from different points in the network continuously changes $[9,10]$. These newly accessible phenotypes can provide fuel for evolution if a new function is required. For example, upon environmental change that alters selection pressures, different members of a genotype network may reveal different adaptive potentials. Thus, it is predicted that evolution can maintain a given molecular phenotype despite mutations, and yet explore new adaptive potential at the same time[11]. These computational results have many implications for the role of mutational robustness and punctuated changes in the evolution of novel structures and functions in general[12]. More specifically, they can guide our understanding of RNA evolution in the laboratory and in natural systems. This article reviews several recent empirical approaches to ribozyme evolution that were aimed at 
understanding how mutational robustness, standing genetic variation and environmental change can promote adaptation[13-15].

\section{Selection for a previously optimized function to investigate genotype networks}

When starting from a molecule of known function, an experiment can be designed to introduce mutations and then select for this already existing function. Most mutations have a deleterious effect on function. Thus, selection for a pre-existing function will be dominated by the purging of deleterious mutations from the population, which is termed purifying selection or negative selection. Whether the starting RNA molecule is the product of natural selection, or laboratory selection, it is fairly likely that it is efficient at this pre-existing function, especially under selection conditions that have been chosen specifically to optimize the activity of this sequence. It is therefore unlikely that sequences with significantly higher relative activity exist within the local mutational neighborhood. Yet, the possibility of improving activity through in vitro selection is always a possibility, especially when an RNA sequence is removed from its natural genetic and cellular context. Typically, laboratory evolution experiments are carried out to find new functions, such as a ribozyme able to catalyze a new reaction. Selecting for a function that already exists may seem counter intuitive, but it can reveal interesting features of the evolving system. Importantly, this type of experiment can be used to explore genotype networks. These experiments are needed to evaluate the extent to which

findings from computational analysis of secondary structures holds true for higher 
levels of complexity, including the native three-dimensional structures required for biological functions. Empirical genotype networks have important implications for the maintenance of RNA functions when mutation rates are high, such as in RNA viruses, attenuated viral vectors [16], mutator strains of bacteria, and lineages of cancer cells. In addition, these genotype networks can also lower the minimum replication fidelity required for life to emerge, a concept known as the phenotypic error threshold $[17,18]$.

\subsection{Selection for reverse-splicing by a self-splicing ribozyme}

To investigate RNA genotype networks in the lab, we previously evolved a naturally occurring ribozyme under purifying selection for its native activity. We chose for this study the Azoarcus group I intron ribozyme. This ribozyme was chosen because it has a very stable structure, and was therefore expected to be robust to mutations, facilitating genotype network exploration. Variants of this ribozyme can be selected in the lab based on their ability to bind an RNA oligonucleotide and transfer the 3'end of the substrate to the 3'-end of the ribozyme (Fig. 1A). This transesterification reaction is the reverse of the second step of the ribozymes natural self-splicing activity. The substrate sequence that becomes attached to the 3'end of the ribozyme was used as a primer-binding site for reverse transcription, allowing selective amplification (PCR, transcription) of only reacted molecules. Molecules without activity, or with very low activity, were purged from the populations (purifying selection). It is worth noting that a molecule could be eliminated from the population because it contains a single mutation that is critical for catalytic activity, 
such as a nucleotide that is highly conserved across known ribozymes[19].

Alternatively, a ribozyme could be selected against because it has several mutations each with a slightly deleterious effect. These two alternatives are analogous to what is termed strong and weak purifying selection at the organismal level[20].

Starting from a DNA template of the wild-type ribozyme sequence, mutations were introduced with error-prone PCR at a rate of 0.0066 per nucleotide per PCR, over a stretch of $156 \mathrm{bp}$ of DNA inside the primer binding sites. Thus, after each selection cycle, most molecules received 1 new mutation, with a probability $\mathrm{P}(1)=$ 0.37 , based on binomial statistics. It was almost as likely that molecules experienced no new mutations $(\mathrm{P}(0)=0.36)$, and molecules with 2-4 new mutations were also reasonably frequent; $\mathrm{P}(2)=0.19, \mathrm{P}(3)=0.06, \mathrm{P}(4)=0.02$. Given the number of molecules used to start each cycle $\left(20\right.$ pmoles $=1 \times 10^{13}$ molecules $)$, we expected that every combination of three or fewer mutations would be present in multiple copies in the initial population, while combinations of four or more mutations would be randomly sampled due to the extremely large number of combinations. From the mutation rate, we expected to both explore new mutations at a reasonable rate, and still maintain selected molecules in the population.

The primers for error-prone PCR also added the $17 \mathrm{nt}$ promoter sequence for T7 RNA polymerase. The starting RNA population was transcribed in vitro and purified by denaturing PAGE. The selection reaction was then set up with 20 pmoles RNA and 100 pmoles oligonucleotide substrate, and the population was allowed to react for $1 \mathrm{~h}$ at $37^{\circ} \mathrm{C}$ in ribozyme reaction buffer. Reacted RNA molecules were reverse-transcribed to cDNA using a primer specific to the 3'-end of the substrate 
oligonucleotide. This cDNA was amplified by PCR with the same reverse-primer, and a forward primer complimentary to the 5'end of the ribozyme, which re-introduced the T7 promoter sequence to the 5'-end. A second PCR was then performed using a nested 3'-primer that removed the substrate sequence and returned the ribozyme to its unreacted catalytic form. This second PCR was carried out under the error-prone conditions. This DNA was transcribed to start the next selection cycle. After each cycle, the cDNA, prior to error-prone PCR, was stored in the freezer. We carried out 10 cycles of selection. We prepare each of the 10 cDNA samples for next generation sequencing by a PCR that added a unique six nucleotides ("barcode", or "index") and the required adaptor sequences for next generation sequencing. The sequencing facility pre-processed the data to remove low quality reads, and separated the sequence data into separate files based on the six-nucleotide index. We also eliminated from analysis sequences that did not cover the full ribozyme sequence.

To analyze the data, we first split the sequencing reads into their corresponding selection cycle based on the barcodes introduced prior to sequencing. Then, for each selection cycle, we used a pairwise alignment of each sequencing read to the wild-type ribozyme, and recorded the position and nucleotide identity of all nucleotide differences. We counted the total number of differences, including indels, for each nucleotide position of the wild-type sequence, and normalized this to the number of reads with that specific barcode. This gave us a "fraction mutated" at each nucleotide position, and the rate of mutation accumulation over ten consecutive selection cycles (see Fig. 1B for example nucleotide positions). We used linear regression analysis to characterize the rate of 
mutation accumulation in two ways. First, to determine if any mutations were under strong selection, we determined if any positions were accumulating mutations faster than expected from the mutation supply rate. We found no such position in our data, confirming that the selection was dominated by purifying selection. Next, to determined which positions could tolerate mutations, we looked for non-zero mutation accumulation. Several positions were found to have accumulated mutations at a rate significantly greater than zero (Fig. 1C). These positions included both peripheral loop regions and some positions involved in base-pairing interactions.

To visualize the genotype network explored by these experiments, we used a network graphing program (Cytoscape). The sequence data was first clustered at 97\% identity (using USEARCH), in an effort to disregard mutations introduced by PCR and sequencing errors. It is important to realize that most clustering algorithms are "greedy" meaning that the order of the sequences in the file will change the consensus sequence or centroid of the cluster and the size of the cluster. Before assigning importance to the consensus, centroid, or size of a cluster, the effect of sequence order should be evaluated. We connected clusters with edges in our graph based on the distance between sequences in the cluster. For example, if a sequence in one cluster had less than 3 mutations from a sequence in another cluster, then the two clusters could be connected by an edge. This cut-off needs to be decided for each experiment based on several factors. The graph should be visually tractable. It is very hard to represent more than $\sim 1000$ data points in any graph. Also, a strict distance $=1$ cutoff often produces many fragmented graphs. For these reasons, the 
details of the experiment and questions need to be carefully considered. It is possible that many intermediate sequences are missing because they have been purged by selection, or because the sample was not sequenced to a sufficient depth.

\section{Mutation without selection to investigate robustness and epistasis}

Experiments that force the accumulation of random mutations in populations of organisms and viruses have been important for understanding the distribution of fitness effects of new mutations, and for identifying systematic interactions between these effects[21-24]. Fitness effects and interactions are equally important at the level of individual genes[25]. However, the fitness landscapes of proteins and RNA molecules encountered by periods of mutation without selection remain poorly understood $[14,26]$. Random mutation accumulation may be a critical source of new functions following gene duplication. Also, due to the pervasive transcription of the non-protein-coding intergenic regions of genomes, it is possible that many transcripts evolve under such conditions. The genotype networks of RNA molecules remains a poorly understood source for the evolution of innovations at the gene level.

Most mutations are deleterious. The random addition of mutations, without filtering by selection for a function, results in the gradual decrease in the activity of a gene. The rate of activity decline is a measurement of mutational robustness. Genes with high mutational robustness would have a slow rate of decline as compared to genes with lower mutational robustness. Also, the addition of one mutation may alter the effect of another mutation, resulting in epistasis. For deleterious mutations, 
the interaction could decrease the effect of additional mutations (antagonistic epistasis), or increase the deleterious effects (synergistic epistasis). Both types of interactions are known to occur. However, if one type of epistasis is more frequent than the other, this can be detected in the shape of the decline in activity. One approach is to fit this activity decline data to the equation $w(n)=\exp \left(-\alpha n^{\beta}\right)[27,28]$. This equation reflects the average deleterious effect in the parameter $\alpha$, and accounts for epistatic interactions in the parameter $\beta$. Values of $\beta<1$ indicate a predominance of antagonistic epistasis, while values of $\beta>1$ indicate a predominance of synergistic epistasis. No epistasis or equal amounts in both directions would be indicated by $\beta=0$.

\subsection{Robustness and epistasis in the Azoarcus ribozyme}

We previously carried out random mutation addition in the Azoarcus ribozyme[14]. To accomplish this, we used error-prone PCR to introduce mutations at a rate of about one mutation per individual per PCR (see Methods). The PCR product was transcribed to RNA for functional analysis. The RNA was also reverse transcribed back to cDNA and subject to an additional error-prone PCR, and this population was transcribed for functional analysis. In this way, several consecutive cycles were performed without selecting for ribozyme activity, creating populations with incrementally increasing numbers of mutations. At each cycle, it was necessary to PAGE purify the RNA to prevent the accumulation of short PCR artifacts.

To accurately count the number of mutations per molecule, samples of each population were analyzed by high-throughput sequencing. The reads from each 
population were aligned to the wild-type reference sequence, and mutations were counted. The ribozyme "fitness" was plotted against the average number of mutations per molecule for a sample of sequences from each cycle. To account for sequencing errors, a "clonal" sample of the wild-type ribozyme was also reverse transcribed and analyzed by sequencing. Mutations in this sample came from DNA library preparation and sequencing. The average number of mutations in this sample was subtracted from each population average prior to fitting the data to the epistasis equation (Fig. 2).

We carried out functional analysis of the populations under varying selection pressures. Relaxed selection pressure was achieved by elevating the amount of magnesium in the reaction buffer. Curve fitting to the equation above demonstrated a relationship between the average deleterious effect of mutations $(\alpha)$ and the epistatic interactions ( $\beta$ ). As magnesium was increased, the average deleterious effect of mutations decreased, and the amount of synergistic epistasis increased (Fig. 2B). Interestingly, the extracted parameters were very similar to previous reports from an analogous set of experiments on a protein enzyme, that also found a predominance of synergistic epistasis, and the same trend in the relationship between $\alpha$ and $\beta$ [26]. In contrast, previous work on simple RNA structures showed predominantly antagonistic epistasis [29]. In combination, the results support the notion that complexity of structure controls the nature of the interaction between deleterious mutations at the gene level, a concept that has been shown at the genomic level [30]. 


\section{Selective sweeps from standing genetic variation}

A classic view of selection involves the introduction of a rare mutation with a very strong effect on fitness that subsequently reaches fixation in the population before the introduction of any other mutation with similar effect on fitness. This is termed a hard selective sweep, which is expected to be the dominant mode of adaptation under what is termed the strong-selection, weak-mutation regime. However, in situations where the effects of beneficial mutations are weak or the frequency of new mutations is relatively frequent, soft selective sweeps would be detected as the simultaneous rise of multiple mutations. Soft sweeps may be a dominant mode of rapid evolution in the lab[31] and in natural populations[32], and may even be the primary mode of recent human evolution[33]. Nevertheless, the debate over the importance of the mutation supply rate in adaptive evolution persists[34], suggesting the need for further experimental evidence under precisely controlled conditions.

\subsection{Monitoring selective sweeps during the evolution of the Azoarcus ribozyme to a} phosphorothioate substrate.

We have monitored the rise in the frequency of mutations in populations of group I intron ribozymes evolving in vitro. For this experiment, we first produced a population with standing genetic variation through multiple rounds of purifying selection, as described above. We then changed the selection pressure by altering the substrate used during selection to contain the more challenging thio-phosphate. We selected for activity on this new substrate for eight rounds of in vitro selection, 
and monitored the evolution as the change in the genetic diversity of the population over time using next generation sequencing.

To detect selective sweeps of mutations, we used a linear regression analysis similar to that described above for detection of purifying selection. However, in this data, we found multiple mutations that were increasing more rapidly than expected from the mutation supply rate (Fig. 3A). We interpreted the rapid rise of a mutation as a sign that it is either beneficial to the ribozyme activity alone, or in combination with other mutations. With several mutations rising simultaneously in the population, we calculated the frequency at which each or the rising mutations cooccurred. For this calculation, we used linkage disequilibrium as a measurement of co-occurrence because this metric also takes into account the individual frequency of each mutation. We found two classes of mutations. Several individual mutations did not co-occur more frequently that expected from their individual frequencies, indicating that they are all rising to higher frequency individually in the population. In addition, we found a group of four mutations that frequently occurred together in the same molecule (marked by asterisk in Fig. 3). We refered to genotypes with these four mutations as $\mathrm{Azo}^{*}$. Interestingly, these mutations individually were not significantly beneficial (Fig. 3B). However, following eight rounds of purifying selection, they co-occurred by chance at a sufficient frequency to enable their selection do to their combined effect on the activity toward the new substrate. These results demonstrate the ability to monitor and interpret soft selective sweeps in population of evolving ribozymes. 


\section{Trajectories and landscapes}

Once an interesting sequence is discovered by selection in the lab, it can be informative to investigate how selection discovered this molecule. This knowledge can inform us about natural evolution, and can guide library designs and selection strategies that will better lead to the selection of desirable molecular properties in the lab. To analyze the different routes to higher fitness, it is necessary to determine the effects of mutations arising in different orders. Each unique ordering of mutations is called an evolutionary trajectory. The different orders of mutations create different increases and decreases in fitness as mutations are added. The trajectories can be used to understand constraints on rates of adaptation, if some trajectories are favored, and others are prohibited. RNA evolution in the laboratory can experimentally test how trajectories constrain evolution theoretically and in practice.

However, between any two sequences, there are many evolutionary trajectories. The number scales as $n$ ! where $n$ is the number of differences between two sequences. For example, if selection produces an RNA molecule of higher fitness that required 8 specific mutational changes, the total number of sequences that could be encountered (the presence or absence of these eight mutations) is $2^{8}=256$. But, there exist $8 !=40,320$ different ways to get across the 256 different genotypes. The reconstruction of evolutionary trajectories has been accomplished by several labs working with RNA[2,15] and DNA enzymes[35], but further effort is required to improve experiments so that they are standardized, sharable, and easier to produce and visualize. 
For complete illucidation of all evolutionary trajectories it is necessary to control the diversity of the starting library. This is because the relative activity of individual sequences, not the fitness effect of individual mutations, needs to be determined. This requires that every sequence appears more than one time in the library. In fact, for high statistical significance, it is recommended that each sequence is present at well over 1000 copies, and is detected by about 1000 reads from a next-generation sequencer. Currently, with the Hi-seq platforms it is possible to obtain $\sim 2$ billion sequences per run, allowing about 2 million sequences to be read at 1000X coverage. This enables analysis of the trajectories between sequences that differ by up to 21 positions, because the number of sequences required to analyze all trajectories is $2^{21}=2.1$ million. It is important to note that this is limited by the actual sequencing platform and the reagents being used. Current Mi-seq platforms are limited to sequences that differ at 14 positions $\left(2^{14}\right.$ sequences $x 1000$ reads per sequence $=16$ million reads). While sequencing throughput continues to improve, the exponential expansion of complexity makes it unlikely that significantly more complex libraries will be feasible in the near future, barring another breakthrough in sequencing technologies. Another approach to overcoming the complexity of sequence space is to focus on only the most active sequences and their immediate mutational neighbors [2]. In fact, it is arguable that natural systems do not exhaustively explore sequence space, and a sampling approach may of high biological relevance.

\subsection{Trajectories to increased reverse-splicing activity}


To analyze the possible evolutionary trajectories encountered in the lab, we made all the sequence intermediates between the wild-type ancestor, and the evolved $A z o^{*}$ variant. We then determined the reverse splicing activity of each variant relative to the wild-type sequence by traditional biochemical techniques (not high-throughput sequencing). By plotting relative activity (y-axis) as a function of added mutations (x-axis), it is possible to compress several trajectories into a single two-dimensional graph (Fig. 4A). To simplify visualization, we have plotted on the same graph all trajectories that begin with the same first mutational step. What is apparent in these trajectories is that three of the four initial mutations are deleterious. Thus three quarters of the trajectories would be highly unlikely under the assumption of strong-selection, weak-mutation[36]. In fact, in our previous experiments, populations that had not accumulated standing genetic variation by genotype network exploration did not discover the $A z o^{*}$ genotype, and adapted more slowly[13].

\subsection{Environmental effects on trajectories}

Next, we compared the same evolutionary trajectories from wild-type to $A z o^{*}$ while changing environmental factors (Fig. 4B). One environmental change involved switching out the native RNA oligonucleotide substrate for a new substrate that contained a chemically challenging thio-phosphate at the cleavage site. The second change involved introducing thermal stress, by adding a denaturant (5M formamide) that destabilizes RNA structures. The landscapes all have similar shapes in the different environments. For example, there are not many crossing trajectories that 
would be expected from very strong gene-by-environment interactions. However, many intermediates on the way to $A z o^{*}$ are only significantly beneficial in one environment (new, red). This is especially true for genotypes that are 2-4 mutations away from the wild-type sequence. Thus, while traditional gene-by-environment interaction plots would not likely uncover a significant interaction effect[37], the effect on the trajectories is expected to be important for adaptation.

The above analysis of trajectories highlights the importance of genotype networks and environmental change in the evolution of innovations at the molecular level. Future work will be aimed at adapting the analysis of trajectories using highthroughput sequencing. Some potentially useful protocols and considerations for this future work is included in the following section (Methods).

\section{Experimental considerations}

\subsection{Library synthesis for evolutionary trajectories}

The library design begins with the alignment of two sequences that represent ancestral and derived RNA molecules. For example, the ancestral sequence could be an inferred node from a phylogenetic tree, and the derived sequence from an extant source. Or, if laboratory evolved ribozymes are under investigation, the ancestral sequence would be the starting wild-type sequence, and the derived sequence would be one found following an in vitro selection experiment. In either case, the investigation is aimed at determining the possible pathways that evolution could have followed. Global pairwise alignment reveals the positions that are different between the ancestral and derived sequences. The most parsimonious conclusion is 
that only these positions changed during the course of natural or laboratory evolution, i.e. there were no back mutations. Each difference can then be written as a single IUPAC ambiguity code that represents two nucleotides. In fact, a single nucleotide sequence, with correct IUPAC codes, can represent all the possible sequences that can be made by the combinations of differences in these positions (if the two molecules are the same length. The issue of indels is considered below). This collection of sequences can then be chemically synthesized on an automated synthesizer, in a single oligonucleotide synthesis. Many companies will accept ambiguity codes as an instruction to add two or more bases during the synthesis of the specified nucleotide.

For example, take the DNA sequences (1) GGEATTAC and (2) $\underline{\mathrm{C} G} \underline{\mathrm{T}} \mathrm{AT} \underline{\mathrm{G}} \mathrm{AC}$. These two sequences differ at three positions, which are underlined. There are $2^{3}=$ 8 combinations of the presence and absence of the sequence changes required to convert sequence (1) into sequence (2). These eight sequences can be represented by the sequence $\underline{\mathrm{S}} \underline{\mathrm{Y} A T} \underline{\mathrm{K} A C}$, where $\mathrm{S}=\mathrm{G}$ or $\mathrm{C}, \mathrm{Y}=\mathrm{C}$ or $\mathrm{T}$, and $\mathrm{K}=\mathrm{G}$ or $\mathrm{C}$. Ordering this sequence, with ambiguity codes, will yield the synthesis of all eight sequences in a single combinatorial synthesis. Note that the different reactivities of each nucleotide phosphoramidite will result in unequal probability of all sequences. Precisely weighed out "hand-mixes" are suggested if near-equal probabilities are required. Also, if insertions or deletions need to be included, a separate synthesis is needed for each collection of sequences with different lengths, such that one indel position will require two separate syntheses, one with the insertion and one without. If the synthetic oligonucleotide also contains the $17 \mathrm{nt}$ promoter for $\mathrm{T} 7$ 
RNA polymerase, it can be transcribed to RNA after being made fully or partially double stranded.

\subsection{Random mutagenesis}

Several mutagenic PCR protocols are available in the literature. The protocol from Cadwell and Joyce is suitable for most RNA applications because it does not favor any particular type of substitution, and distributes mutations evenly throughout the sequence [38]. In addition, the mutation rate can be controlled to some extent by the amount of template DNA input and number of PCR cycles used, with more amplification creating more mutations [39]. The standard protocol produces a mutation rate of 0.0066 mutations per molecule per PCR. This rate results in an average of 1 mutation per molecules for molecules that are $151 \mathrm{nt}$ long. For longer sequences it may be desirable to lower the mutation rate slightly by starting with higher template and reducing the total PCR amplification. Other protocols can achieve different mutation rates. It is important to remember that mutations are only introduced between primer binding sites. If mutations are desired in the entire RNA, adapters can be added to the DNA template, but care should be taken to confirm that if these adapters are transcribed, the structure and activity of the RNA is not changed [40]. For molecules that will be transcribed by T7 RNA polymerase, the 17 b.p. promoter sequence can be used as a primer binding site. However, the Tm of this sequence is low $\left(\sim 40^{\circ} \mathrm{C}\right)$, and it is recommended that $\sim 6-12$ additional base pairs are added to the $5^{\prime}$-end to match the Tm of the other primer. A restriction site is a common choice because it can facilitate cloning if desired. 


\subsection{RNA preparation for sequencing}

For high throughput sequencing, it is necessary to convert RNA to cDNA and add sequencing adapters for appropriate sequencing platforms. It is possible to do this in either order, because adapters can be ligated at the RNA stage, or at the DNA stage. When starting from a known ribozyme sequence, reverse transcription is carried out with a sequence specific primer. The same primer used in mutagenesis is typically used. For the experiments discussed here, we assume that the starting sequence is known, and can be used to design primers for reverse transcription and PCR.

\subsubsection{Ligation-type ribozyme reactions}

Several types of ribozymes can attach or ligate a substrate sequence to either the $5^{\prime}$ or 3' end of the ribozyme sequence. For example, Group I and Group II self-splicing introns can catalyze reverse-splicing reactions that attach a portion of an exon analogue oligonucleotide to their 3'-end. Small self-cleaving ribozymes can also be made to ligate appropriate substrates in a reverse cleavage reaction[41]. Also, several classes of non-natural ligase ribozymes have been discovered that attach substrate oligos to their 3'-end. For all these "ligation" reactions, it is convenient to use this newly attached sequence for selection. Molecules can be reverse transcribed with a primer specific to the 3 '-end of the RNA. This cDNA is subsequently PCR amplified with one primer specific to the ribozyme, and the other primer specific to the ligated substrate to achieve selective amplification of reacted 
molecules (see Fig 1. for example). This approach amounts to amplicon sequencing that is similar to $16 \mathrm{~S}$ metagenomic sequencing, for example. A common approach is to use sequence specific promoters in a first PCR, and then add the sequencing adapters in a second PCR. It is recommended to use hi-fidelity enzymes throughout, and limit the number of PCR cycles used to avoid PCR based recombination [42].

\subsubsection{Self-cleaving ribozymes}

Many naturally occurring ribozymes catalyze intramolecular RNA cleavage reactions. It is possible to detect this activity by sequencing because the RNA molecule, and the DNA sequencing read, become shorter upon reaction. The scissile phosphate bond is often near the $5^{\prime}$-end of the construct, and therefore it is possible to sequence the majority of the ribozyme in the 3'-product fragment. If care is take to only introduce sequence variation downstream of this cleavage site, then no information is lost during sequencing. Each read of a sequencer reports on an observation of a specific sequence and the length indicates if it is in a reacted or unreacted form. If library complexity is controlled, hundreds to thousands of observations of each individual sequence is possible, leading to strong statistical significance.

Again, it is possible to ligate sequence adapters to either RNA or cDNA. For RNA ligation, care must be taken to ensure that the $5^{\prime}$-end of the RNA is a substrate for RNA ligase. For example, protocols with T4 RNA ligase require that triphosphorylated RNA is first treated with alkaline phosphatase to remove the triphosphate, and subsequently with T4 polynucleotide kinase to add a 
monophosphate. In many cases, quantitative conversion is inconsistent, or not possible. RNA ligase also has known sequence biases. Alternatively, several 5'-RACE (Rapid Amplification of cDNA Ends) protocols can also be used to maintain the sequence information at the 5'-end of the RNA. This has the advantage of quickly converting RNA to cDNA to avoid further ribozyme cleavage, and requires no alterations to the RNA before reverse transcription. Several 5'-RACE protocols rely on non-templated nucleotides being added to the 3'-end of the cDNA. This nontemplated addition can be carried out by polynuclotide transferase or, in some cases, by the reverse-transcription enzyme itself. The non-templated nucleotides are used for second strand synthesis with a primer that matches the non-templated sequence. A fixed primer-binding site can be added to this second strand synthesis oligo. This fixed sequence can be either the sequencing adapter itself, or a primerbinding site to add adapters in a subsequent PCR. This latter strategy has the advantage of working with commercial indexing primers, such as those designed for Illumina Nextera kits. This is advantageous for resource sharing between projects and labs.

Acknowledgements This work has been supported by the National Science Foundation, grant number MCB-1413664. Thanks to all the co-authors that contributed to the research summarized in this work. Special thanks to Andreas Wagner for the many constructive conversations that shaped the ideas presented in this article. Another special thanks to Evandro Ferrada and Rhiddhiman Dhar for developing and assisting in data analysis approaches.

[1] N. Lehman, G.F. Joyce, Evolution in vitro: analysis of a lineage of ribozymes, Curr. Biol. 3 (1993) 723-734. doi:10.1016/0960-9822(93)90019-K.

[2] J.I. Jiménez, R. Xulvi-Brunet, G.W. Campbell, R. Turk-MacLeod, I.A. Chen, Comprehensive experimental fitness landscape and evolutionary network for 
small RNA, Proc. Natl. Acad. Sci. 110 (2013) 14984-14989. doi:10.1073/pnas.1307604110.

[3] J.N. Pitt, A.R. Ferre-D'Amare, Rapid construction of empirical RNA fitness landscapes, Science. 330 (2010) 376-379. doi:10.1126/science.1192001.

[4] M.J. Wiser, N. Ribeck, R.E. Lenski, Long-term dynamics of adaptation in asexual populations, Science. 342 (2013) 1364-1367.

[5] L.W. Ancel, W. Fontana, Plasticity, evolvability, and modularity in RNA, J. Exp. Zool. 288 (2000) 242-283. doi:10.1002/1097010X(20001015)288:3<242::AID-JEZ5>3.0.C0;2-0.

[6] P. Schuster, W. Fontana, P.F. Stadler, I.L. Hofacker, From sequences to shapes and back: a case study in RNA secondary structures, Proc. Biol. Sci. 255 (1994) 279-284. doi:10.1098/rspb.1994.0040.

[7] A. Wagner, The molecular origins of evolutionary innovations, Trends Genet. 27 (2011) 397-410. doi:10.1016/j.tig.2011.06.002.

[8] T. Jörg, O.C. Martin, A. Wagner, Neutral network sizes of biological RNA molecules can be computed and are not atypically small, BMC Bioinformatics. 9 (2008) 464. doi:10.1186/1471-2105-9-464.

[9] W. Fontana, P. Schuster, Continuity in evolution: on the nature of transitions, Science. 280 (1998) 1451-1455.

[10] A. Wagner, Robustness and evolvability: a paradox resolved, Proc. Biol. Sci. 275 (2008) 91-100. doi:10.1098/rspb.2007.1137.

[11] A. Wagner, Neutralism and selectionism: a network-based reconciliation, Nat Rev Genet. 9 (2008) 965-974. doi:10.1038/nrg2473.

[12] A. Wagner, Robustness and Evolvability in Living Systems, Princton University Press, 2005.

[13] E.J. Hayden, E. Ferrada, A. Wagner, Cryptic genetic variation promotes rapid evolutionary adaptation in an RNA enzyme, Nature. 474 (2011) 92-95. doi:10.1038/nature10083.

[14] E.J. Hayden, D.P. Bendixsen, A. Wagner, Intramolecular phenotypic capacitance in a modular RNA molecule, Proc. Natl. Acad. Sci. 112 (2015) 12444-12449. doi:10.1073/pnas.1420902112.

[15] E.J. Hayden, A. Wagner, Environmental change exposes beneficial epistatic interactions in a catalytic RNA, Proc. R. Soc. B Biol. Sci. 279 (2012) 3418-3425. doi:10.1098/rspb.2012.0956.

[16] P. Ketzer, S.F. Haas, S. Engelhardt, J.S. Hartig, D.M. Nettelbeck, Synthetic riboswitches for external regulation of genes transferred by replicationdeficient and oncolytic adenoviruses, Nucleic Acids Res. 40 (2012) e167-e167. doi:10.1093/nar/gks734.

[17] N. Takeuchi, P.H. Poorthuis, P. Hogeweg, Phenotypic error threshold; additivity and epistasis in RNA evolution, BMC Evol. Biol. 5 (n.d.) 9-9. doi:10.1186/14712148-5-9.

[18] Á. Kun, M. Santos, E. Szathmáry, Real ribozymes suggest a relaxed error threshold, Nat. Genet. 37 (2005) 1008-1011. doi:10.1038/ng1621.

[19] J.J. Cannone, S. Subramanian, M.N. Schnare, J.R. Collett, L.M. D'Souza, Y. Du, B. Feng, N. Lin, L.V. Madabusi, K.M. Müller, N. Pande, Z. Shang, N. Yu, R.R. Gutell, The comparative RNA web (CRW) site: an online database of comparative 
sequence and structure information for ribosomal, intron, and other RNAs, BMC Bioinformatics. 3 (2002) 2.

[20] B.H. Good, M.M. Desai, The equivalence between weak and strong purifying selection, ArXiv12104500 Q-Bio. (2012). http://arxiv.org/abs/1210.4500 (accessed May 13, 2016).

[21] S.F. Elena, R.E. Lenski, Epistasis between new mutations and genetic background and a test of genetic canalization, Evol. Int. J. Org. Evol. 55 (2001) 1746-1752.

[22] C.L. Burch, L. Chao, Epistasis and its relationship to canalization in the RNA virus phi 6, Genetics. 167 (2004) 559-567. doi:10.1534/genetics.103.021196.

[23] S.F. Elena, R.E. Lenski, Test of synergistic interactions among deleterious mutations in bacteria, Nature. 390 (1997) 395-398.

[24] C. Haag-Liautard, M. Dorris, X. Maside, S. Macaskill, D.L. Halligan, B. Charlesworth, P.D. Keightley, Direct estimation of per nucleotide and genomic deleterious mutation rates in Drosophila, Nature. 445 (2007) 82-85.

[25] M. Soskine, D.S. Tawfik, Mutational effects and the evolution of new protein functions, Nat. Rev. Genet. 11 (2010) 572-582. doi:10.1038/nrg2808.

[26] S. Bershtein, M. Segal, R. Bekerman, N. Tokuriki, D.S. Tawfik, Robustnessepistasis link shapes the fitness landscape of a randomly drifting protein, Nature. 444 (2006) 929-932. doi:10.1038/nature05385.

[27] C.O. Wilke, C. Adami, Interaction between directional epistasis and average mutational effects, Proc. Biol. Sci. 268 (2001) 1469-1474. doi:10.1098/rspb.2001.1690.

[28] R.E. Lenski, C. Ofria, T.C. Collier, C. Adami, Genome complexity, robustness and genetic interactions in digital organisms, Nature. 400 (1999) 661-664.

[29] C.O. Wilke, R.E. Lenski, C. Adami, Compensatory mutations cause excess of antagonistic epistasis in RNA secondary structure folding, BMC Evol. Biol. 3 (2003) 3. doi:10.1186/1471-2148-3-3.

[30] R. Sanjuán, S.F. Elena, Epistasis correlates to genomic complexity, Proc. Natl. Acad. Sci. U. S. A. 103 (2006) 14402-14405. doi:10.1073/pnas.0604543103.

[31] M.-C. Lee, C.J. Marx, Synchronous waves of failed soft sweeps in the laboratory: remarkably rampant clonal interference of alleles at a single locus, Genetics. 193 (2013) 943-952. doi:10.1534/genetics.112.148502.

[32] P.W. Messer, D.A. Petrov, Population genomics of rapid adaptation by soft selective sweeps, Trends Ecol. Evol. 28 (2013) 659-669. doi:10.1016/j.tree.2013.08.003.

[33] R.D. Hernandez, J.L. Kelley, E. Elyashiv, S.C. Melton, A. Auton, G. McVean, G. Sella, M. Przeworski, others, Classic selective sweeps were rare in recent human evolution, Science. 331 (2011) 920-924.

[34] J.D. Jensen, On the unfounded enthusiasm for soft selective sweeps, Nat. Commun. 5 (2014) 5281. doi:10.1038/ncomms6281.

[35] R. Gysbers, K. Tram, J. Gu, Y. Li, Evolution of an Enzyme from a Noncatalytic Nucleic Acid Sequence, Sci. Rep. 5 (2015) 11405. doi:10.1038/srep11405.

[36] D.M. Weinreich, N.F. Delaney, M.A. Depristo, D.L. Hartl, Darwinian evolution can follow only very few mutational paths to fitter proteins, Science. 312 (2006) 111-114. doi:10.1126/science.1123539. 
[37] E.J. Hayden, S. Bratulic, I. Koenig, E. Ferrada, A. Wagner, The effects of stabilizing and directional selection on phenotypic and genotypic variation in a population of RNA enzymes, J. Mol. Evol. 78 (2014) 101-108. doi:10.1007/s00239-013-9604-x.

[38] R.C. Cadwell, G.F. Joyce, Randomization of genes by PCR mutagenesis., Genome Res. 2 (1992) 28-33. doi:10.1101/gr.2.1.28.

[39] D.S. Wilson, A.D. Keefe, Random mutagenesis by PCR, Curr. Protoc. Mol. Biol. (2001) 8-3.

[40] K.A. Wilkinson, E.J. Merino, K.M. Weeks, Selective 2'-hydroxyl acylation analyzed by primer extension (SHAPE): quantitative RNA structure analysis at single nucleotide resolution, Nat. Protoc. 1 (2006) 1610-1616. doi:10.1038/nprot.2006.249.

[41] A.R. Ferré-D’Amaré, W.G. Scott, Small self-cleaving ribozymes, Cold Spring Harb. Perspect. Biol. 2 (2010) a003574.

[42] W. Yu, K.J. Rusterholtz, A.T. Krummel, N. Lehman, Detection of high levels of recombination generated during PCR amplification of RNA templates, BioTechniques. 40 (2006) 499-507.

\section{FIGURE LEGENDS}

Figure 1. Exploration of genotype networks during selection for pre-existing functions. (A) Selection scheme for group I intron reverse splicing. The ribozyme population is produced by error-prone PCR and in vitro transcription. The ribozyme reaction used $\sim 10^{13}$ molecules and excess substrate (grey line). A substrate specific primer (grey arrow) is used to convert only reacted molecules to DNA in a reverse transcription PCR (RT-PCR). A second PCR uses primers specific to the 5'-end of the ribozyme to produce amplicons in the active form, and add the T7 promoter for subsequent transcription. (B) Nucleotide positions that showed different patterns of mutation accumulation under purifying and directional selection. Sequencing reads were aligned to the wild-type reference sequence. At each nucleotide position, the frequency of any mutation was counted. Above each nucleotide position in the plot are multiple bar graphs, each reporting the frequency of a mutation at that position after a subsequent round of selection. The frequency of mutation for 10 consecutive rounds of selection are shown. The grey bars represent the expected frequency of mutations based on the mutation supply rate during error-prone PCR. Linear regression analysis was used to determine if the rate of mutation accumulation at each site was significantly different than the mutation supply rate. (C) Nucleotide positions with non-zero mutation accumulation were mapped onto the crystal structure of the Azoarcus ribozyme, by replacing the b-factor column with $\mathrm{P}$ value from regression analysis, with red being lowest $\mathrm{P}$-values, and blue higher than $\mathrm{P}=$ 0.05. (D) Networks produced from selected sequences. Each node represents genotypes determined by clustering several sequences at a desired similarity cutoff (i.e. 97\%). Edges connect nodes if the within the two nodes are sequences that differ by less than a specified edit distance. The green node represents the starting wildtype sequence. Nodes with white or blue centers came from separate selections. 
Nodes with a yellow border contain a specific set of four mutations with high cooccurrence. Edges to these nodes are yellow. Panels A-C were previously published in the main text or supporting information of [13].

Figure 2. Robustness and directional epistasis from mutation accumulation. (A-B) Mutations were introduced during several consecutive rounds of error-prone PCR. After each round, the activity of the population was determined relative to a wildtype clone (y-axis). Mutations per molecule were determined by pairwise alignment of sequencing reads to the wild-type reference. The decline in activity was fit to the model $\mathrm{w}(n)=\exp (-\alpha n \beta)$ by a nonlinear least squares method (Matlab). The model allows for an average deleterious effect of mutations $(\alpha)$, and an epistatic interaction between these effects $(\beta)$. Data points represent the average of three technical replicates. Parameters are reported with 95\% confidence intervals of the fit parameter. (C) Altering the magnesium concentration during selection $(2 \mathrm{mM}, 10$ $\mathrm{mM}, 25 \mathrm{mM} \mathrm{MgCl} 2$ ) alters the stringency of selection and reveals a relationship between $\alpha$ and $\beta$. A predominance of synergistic interactions between mutational effects is indicated by $\beta>1$, and no predominant interaction by $\beta=1$. Higher values of $\alpha$ indicate a greater average deleterious effect of random mutations. Deleterious mutation effects are buffered by high magnesium concentrations, but revealed at low magnesium concentrations. This data was published as supporting information in a previous publication [14].

Figure 3. Observing selective sweeps during evolution toward a non-optimized function. (A) Molecules were selected for increased relative activity with a modified substrate. Ribozymes enriched during this selection were sequenced. Sequencing reads were aligned to the wild-type reference allowing identification of specific mutations. The population frequency of specific mutations was determined for several consecutive rounds of selection, and for every nucleotide position in the reference sequence. Linear regression analysis was used to determine mutations that accumulated significantly faster than expected from the mutation supply rate, which are plotted. The yellow asterisks indicate four mutations with high cooccurrence, determined by linkage analysis. (B) The four mutations improve activity relative to the wild-type together, but each individually does not. The relative acitivy of each mutation when added to the wild-type background is plotted. (C) The selected mutations are mapped onto the crystal structure of the Azoarcus ribozyme. The inset shows the proximity of the co-occuring mutations, two of which form a base-pair in the RNA structure. The data in panels A and B were contained with the figures of the supporting information of a previous publication[13]. Panels $C$ was previously published[15].

Figure 4. Detecting multiple trajectories to higher fitness and changes caused by environmental factors. (A) All mutational intermediates between the wild-type ribozyme and a four-mutation variant with higher activity $\left(\mathrm{AzO}^{*}\right)$ were synthesized and their fitness (y-axis) was determined as the activity of this variant relative to the wild-type ribozyme. Each color represents a different order of mutations creating different "peaks" and "valleys" in the fitness trajectory. Each graph starts 
with the same first mutation, as indicated above this data point. (B) Changing environmental factors alters a given trajectory to higher fitness. The fitness (y-axis) is shown as mutations ( $\mathrm{x}$-axis) are added one by one to traverse the fitness landscape from wild-type (left) to the $A z o^{*}$ variant (right). Each graph shows the same order of introduced mutations, but with different altered environmental factors. The native data (blue) shows fitness with an RNA oligonucleotide substrate. The new data (red) shows fitness with a substrate that has a phosphorothioate at the scissile phosphate. The thermal stress data shows fitness in when RNA denaturant (5M formamide) is added to the buffer used for the fitness assay. This data has been published as supporting information in another publication [15]. 

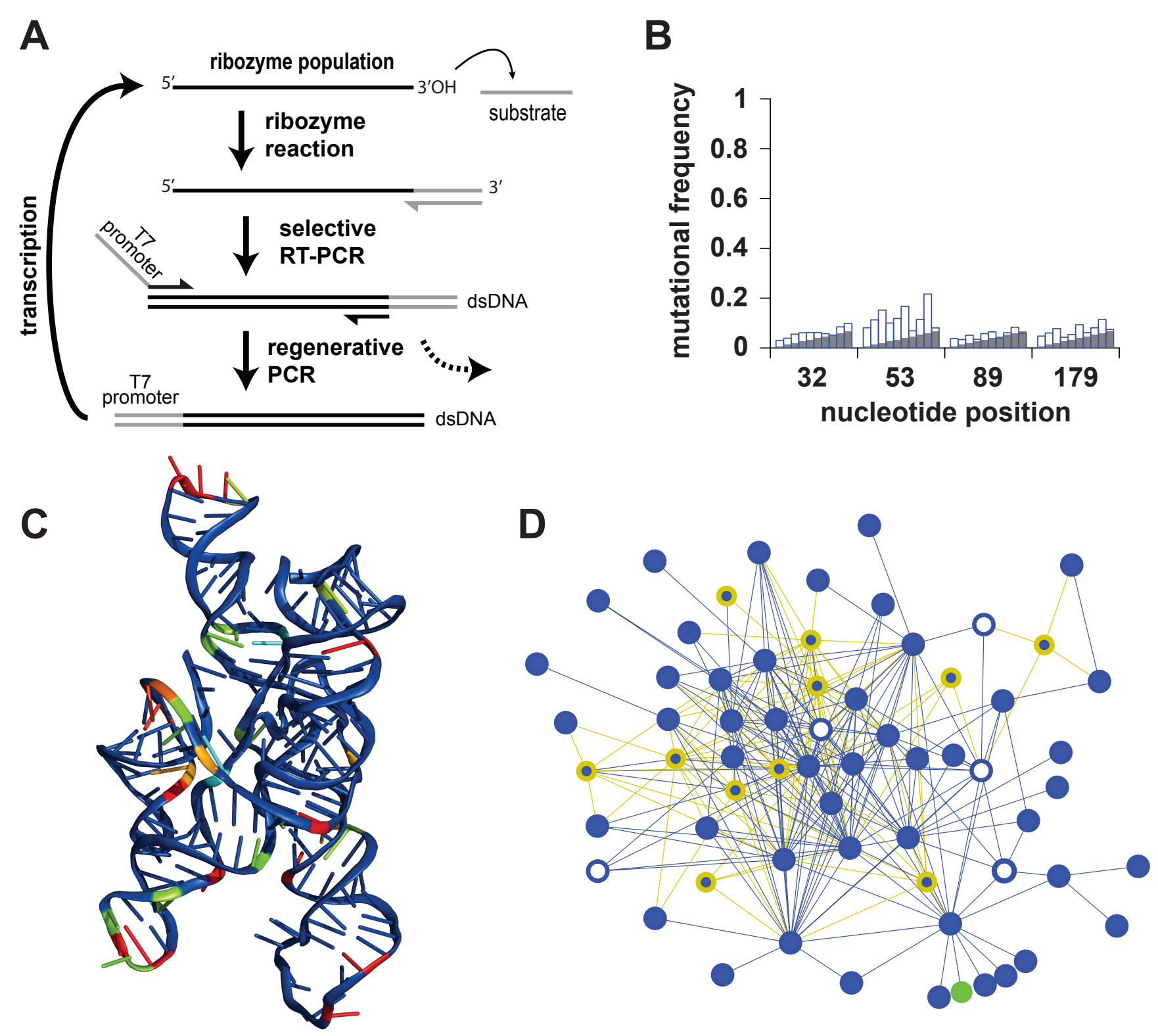
Figure 2
$\mathrm{~A}$

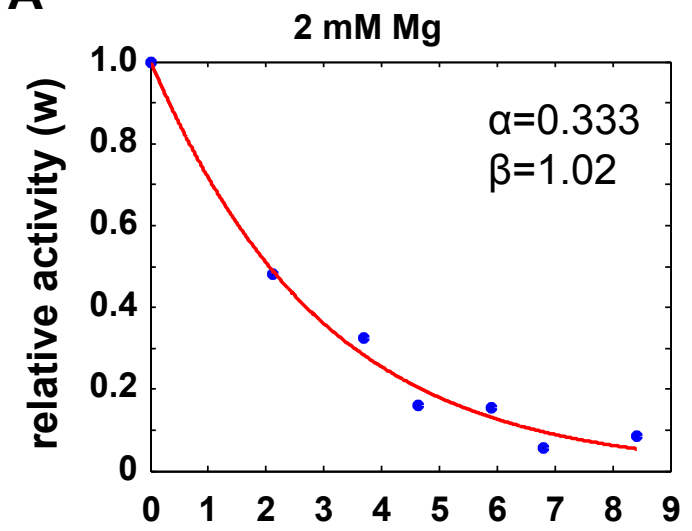

mutations per molecule (n)
B

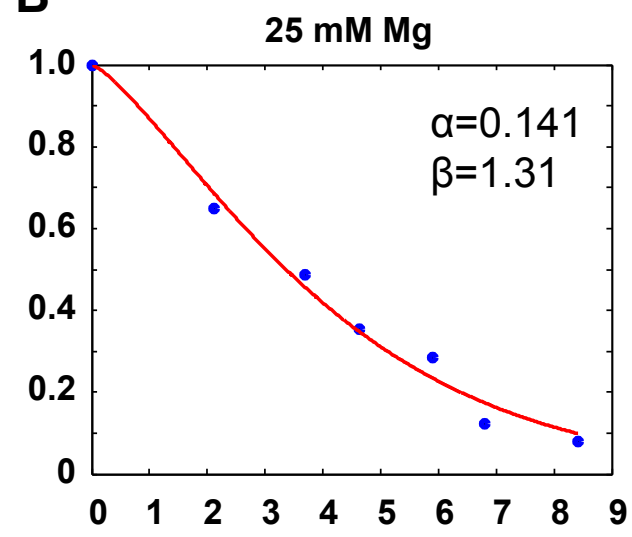
mutations per molecule (n)
C

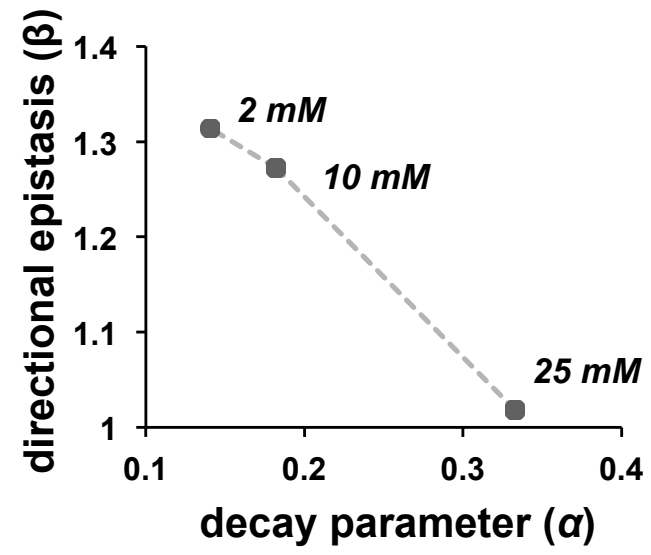


A
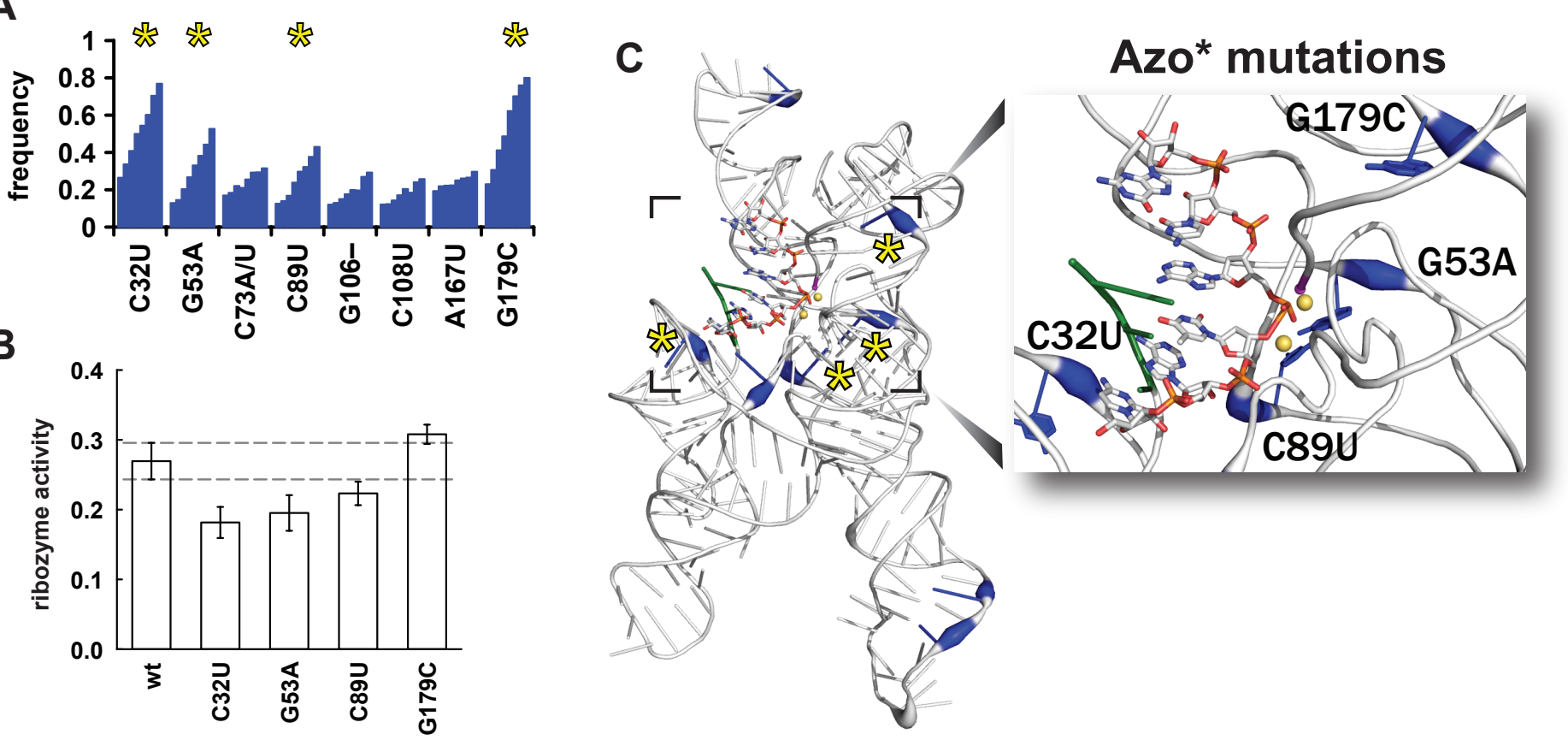
A
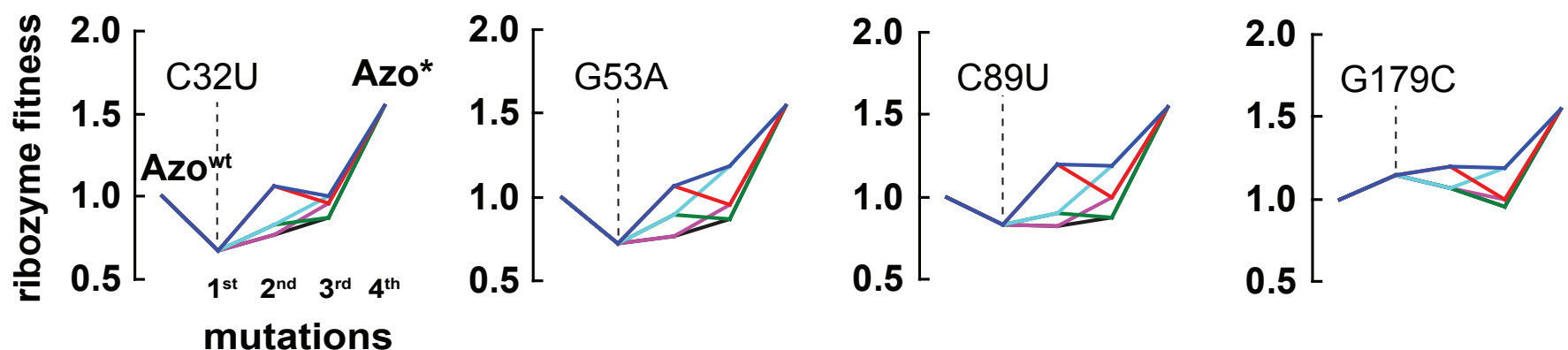

B
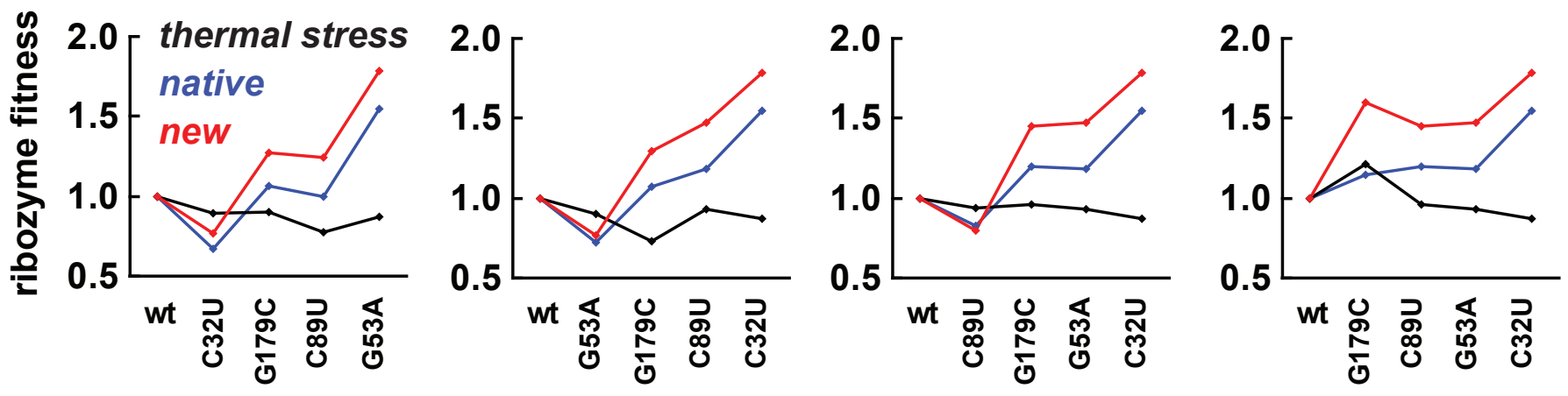